\title{
Fuzzy Multiobjective Traffic Light Signal Optimization
}

\author{
N. Shahsavari Pour, H. Asadi, and M. Pour Kheradmand \\ Department of Industrial Engineering, Science and Research Branch, Islamic Azad University, Kerman, Iran \\ Correspondence should be addressed to H. Asadi; hamed_assadi2000@yahoo.com
}

Received 18 September 2012; Revised 26 March 2013; Accepted 9 April 2013

Academic Editor: Zhiping Qiu

Copyright (C) 2013 N. Shahsavari Pour et al. This is an open access article distributed under the Creative Commons Attribution License, which permits unrestricted use, distribution, and reproduction in any medium, provided the original work is properly cited.

Traffic congestion is a major concern for many cities throughout the world. In a general traffic light controller, the traffic lights change at a constant cycle time. Hence it does not provide an optimal solution. Many traffic light controllers in current use are based on the "time-of-the-day" scheme, which use a limited number of predetermined traffic light patterns and implement these patterns depending upon the time of the day. These automated systems do not provide an optimal control for fluctuating traffic volumes. In this paper, the fuzzy traffic light controller is used to optimize the control of fluctuating traffic volumes such as oversaturated or unusual load conditions. The problem is solved by genetic algorithm, and a new defuzzification method is introduced. The performance of the new defuzzification method (NDM) is compared with the centroid point defuzzification method (CPDM) by using ANOVA. Finally, an illustrative example is presented to show the competency of proposed algorithm.

\section{Introduction}

Delays in urban road networks depend largely on the performance of the networks' signalled Intersections. Traffic congestion is one of the challenging problems in many countries. Traffic congestion wastes a huge portion of the national income for fuel and traffic-related environmental and socioeconomic problems. One of the major problems is the oversaturated network conditions, especially during peak hours.

In urban networks the flow is controlled by traffic lights, and traffic engineers are often faced with the question if the capacity of the networks is exploited by the chosen control strategy. One possible method to answer such questions could be the use of vehicular traffic models in control systems as well as in the planning and design of transportation networks. For almost half a century there were strong attempts to develop a theoretical framework for traffic science.

As is known to all, the main places responsible for traffic congestion are urban intersections, and the primary reason for traffic congestion in urban intersections is the irrational cycle time of traffic lights. In order to separate the conflict of the traffic flow effectively and improve traffic capacity, how to assign the red and green time in a cycle is obviously important.
Generally, longer cycle time leads to greater traffic capacity, but traffic queue and number of pedestrians also increase with increasing the cycle time. In other words, when the saturation of an intersection is small enough, the increase of the cycle time does not go far enough towards traffic capacity, and it only leads to the increase of traffic queue and number of pedestrians.

Even though Bayley [1] showed that long cycle times have negative impacts on pedestrian delays, current policies for traffic signal control still consider primarily vehicle delays. Noland $[2,3]$ showed with London data for small networks that extending pedestrian green times could reduce total delays at a junction. Traffic signals generally aim at minimizing average vehicle delays, while pedestrian delays are not taken into account. Such strategy is reasonable for rural areas or highways where very few pedestrians interfere with vehicular traffic. However, in a central business district with a lot of pedestrians walking around, the strategy that optimizes only vehicle flows would not be suitable because the pedestrian delays are ignored. Ignoring pedestrian delays might even result in people choosing to use vehicles more frequently than walking. So, it is reasonable that pedestrian signal plans be also optimized. 
In this paper, the pedestrians' waiting time does not have any costs for the system, but reducing it will result in increase of the citizens' satisfaction.

Brockfeld and his colleagues [4] focused on global traffic light control strategies and tried to find optimal model parameters in order to maximize the network flows. Gradinescu and his colleagues [5] studied traffic control system using wireless communications, where the vehicles send information to the nearby traffic control system, and then the system will optimize the traffic lights based on the received information. Teo et al. [6] used genetic algorithm to optimize the total green time of all traffic lights at intersections and split of the traffic flows.

Webster [7] pioneered the use of computer simulation to analyse traffic patterns and chose to set traffic light cycle length and cycle splits to improve traffic flow. Yang [8] developed an effective procedure to optimize intersection signal timing by minimizing total delay for both vehicles and pedestrians.

Dong et al. [9] developed simulated annealing particle swarm optimization (Sa-PSO) algorithm based on particle swarm optimization (PSO) and simulation was carried out in a nine-intersection network, and the result shows that the use of Sa-PSO method can reduce $41.0 \%$ of the average delay per vehicle and $30.6 \%$ of the average stop rates compared with fixed time plans. But the research of Sa-PSO was just at the beginning; there were still so much problems for further study.

Also there are certain systems that take advantage of sensors to detect the incoming vehicles and use fuzzy theory to make decisions for extending the green light periods to let more vehicles pass through [10]. Favilla et al. [11] applied fuzzy theory to control of two-way streets. The number of vehicles that have already passed during green time and the length of the traffic queue during red time are used as inputs to the fuzzy rules; the output of the rules is the amount of extension to be applied to the current signal state. They also proposed additional strategies for adopting the numerical bounds on the input and output variables. The concept of pedestrian progression was also proposed in some researches.

The average pedestrian delays model is proposed in "Pedestrian compliance effects on signal delay" by Virkler, based on the assumption that all pedestrians arrive randomly, which means pedestrians who arrive in green enter the intersection without any delay, and pedestrian flow arrives uniformly in red. It is also assumed in the model that the cycle length is constant, and no pedestrian actuation is applied in the intersection [12].

Chilukuri and Virkler [13] proved that the pedestrian delays calculation which assumes random arrivals might not be accurate in a coordinated arterial. Bhattacharya and Virkler [14] studied cyclic flow profiles generated from arrival patterns and developed a method to estimate the delay from the offset with respect to the upstream signal cycle. Then, they used the method to determine favorable signal offsets for pedestrian progression.

In this paper, we present a new fuzzy multiobjective mathematical model to minimize the length of traffic queue and pedestrian delays.

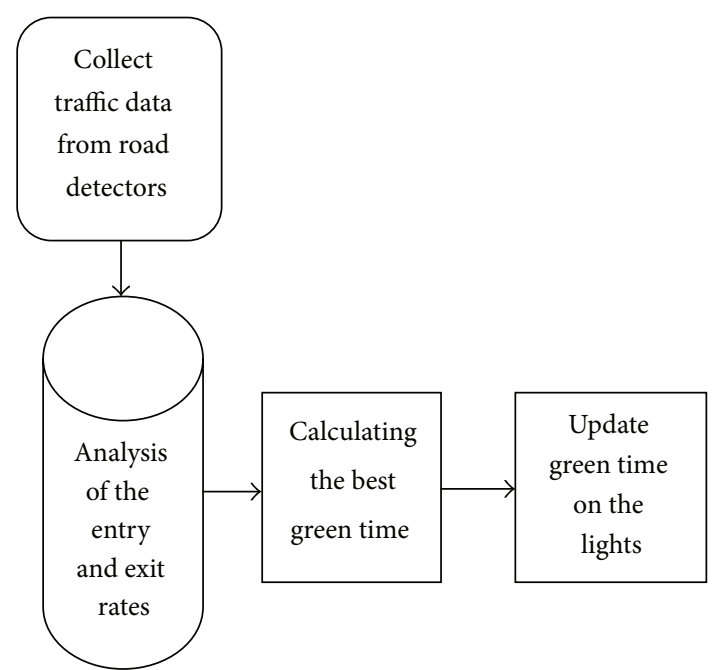

FIGURE 1: Scheme of traffic optimization process using data collected from road detectors.

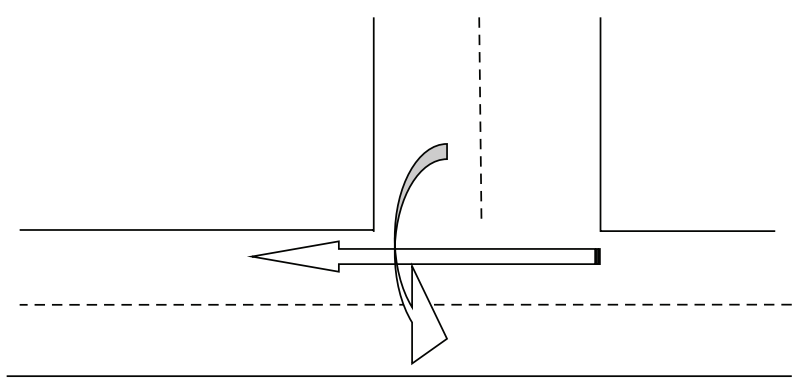

Figure 2: A "two-phase intersection."

The paper is organized as follows. In Section 2, the problem definition is provided. In Section 3 a new defuzzification method is introduced. This is followed by the problem formulation in Section 4. An illustrative example is provided in Section 5; then, in Section 6, experimental evaluation is provided to show the competency of algorithm. Section 7 then concludes this paper.

\section{Problem Description}

The problem discussed in this paper is minimizing the queue length on each side of the intersection during peak hours with considering the entrance and exit rates of each side of the intersection as fuzzy rates.

This study is to calculate green time duration in order to minimize traffic jam length. Before beginning the optimization process, road detector should gather related data of intersection traffic. This data are required to make a decision if current conditions are sufficient or they have to be improved (see Figure 1).

Queue length is the total number of vehicles that line up in front of the intersection waiting to be given the permission for passing the intersection. At each intersection, the side that the traffic flows in is called "phase." For example, in Figure 2 there are two phases at intersection. 
Amber is the signal appearing between the red and green signal, indicating a warning to the traffic flow to slow down as the green signal is going to be ended.

At first, a sensitivity analysis is done on the traffic light at a two-phase intersection, and then it is developed for a fourphase traffic problem.

When phase I has red time (T1), traffic jam length in this phase has an increasing rate. On the contrary, traffic jam length of phase II-experiencing green time-has a decreasing rate. Red time in each phase is sum of green time in other phases which increases as green time devoted to each phase increases. Therefore, jam length in other phases may also increase.

The pedestrians' queue length has an incremental rate and at the end of the green time reaches its highest level (points A1 and A2). As soon as intersection's red time starts, all pedestrians cross the intersection immediately. As a result, pedestrians' queue is equal to zero (see Figure 3).

2.1. Fuzzy Entry and Exit Rate. Since entrance/exit rate of traffic jams at an intersection in a traffic network is uncertain, it is necessary to consider it in an uncertainty condition. One of the best tools to take this uncertainty into account is fuzzy theory.

Definition 1 (fuzzy sets and membership functions). If $X$ is a collection of objects denoted generically by $x$, then a fuzzy set $A$ in $X$ is defined to be a set of ordered pairs $A=\left\{\left(x, \mu_{\widetilde{A}}(x)\right) \mid\right.$ $x \in X\}$, where $\mu_{\widetilde{A}}(x)$ is called the membership function for the fuzzy set. The membership function maps each element of $X$ to a membership value between 0 and 1 .

Definition 2. The triangular fuzzy numbers can be denoted as $\widetilde{A}=\left(a_{1}, a_{2}, a_{3}\right)$, where $a_{2}$ is the central value (if $\mu_{\widetilde{A}}\left(a_{2}\right)=$ 1 then $\widetilde{A}$ is a normal fuzzy number) (see Figure 4). The membership function of such triangular fuzzy number is defined as

$$
\mu_{\widetilde{A}}(x)= \begin{cases}0 & x \leq a_{1}, \\ \frac{x-a_{1}}{a_{2}-a_{1}} & a_{1}<x \leq a_{2}, \\ \frac{a_{3}-x}{a_{3}-a_{2}} & a_{2}<x \leq a_{3}, \\ 0 & x>a_{3} .\end{cases}
$$

Let $\widetilde{A}$ and $\widetilde{B}$ be two triangular fuzzy numbers (TFN) parameterized by the triplets $\left(a_{1}, a_{2}, a_{3}\right)$, and $\left(b_{1}, b_{2}, b_{3}\right)$ respectively, then the operational rules of these two triangular fuzzy numbers are as follows:

$$
\begin{gathered}
\widetilde{A}(+) \widetilde{B}=\left(a_{1}+b_{1}, a_{2}+b_{2}, a_{3}+b_{3}\right), \\
\widetilde{A}(-) \widetilde{B}=\left(a_{1}-b_{1}, a_{2}-b_{2}, a_{3}-b_{3}\right), \\
\widetilde{A}(\times) \widetilde{B}=\left(a_{1} \cdot b_{1}, a_{2} \cdot b_{2}, a_{3} \cdot b_{3}\right) .
\end{gathered}
$$

Entrance/exit rate is considered in terms of triangular fuzzy numbers, and a new defuzzification method is presented for division of two fuzzy numbers.

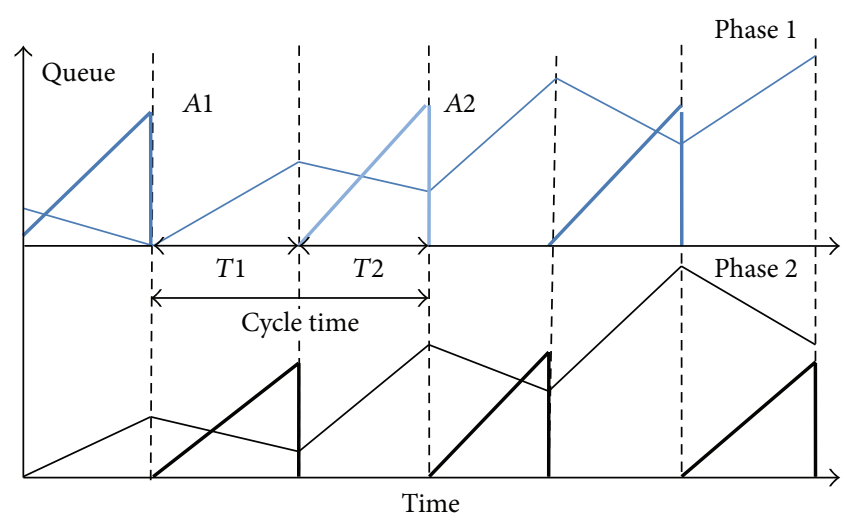

FIGURE 3: Sensitivity analysis for two-phase intersection.

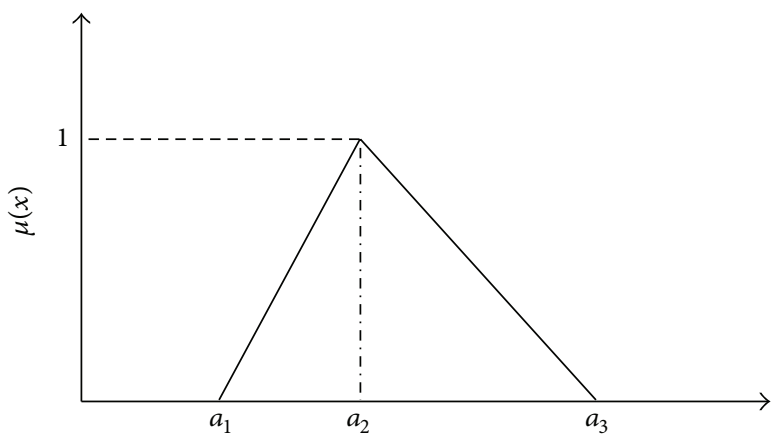

Figure 4: A triangular fuzzy number $\widetilde{A}$.

\section{New Defuzzification Method}

Defuzzification and ranking fuzzy numbers are complex and challenging. This is because fuzzy numbers, usually represented by possibility distributions, can often overlap each other in many practical situations. It is difficult, if not impossible, to clearly determine whether one fuzzy number is larger or smaller than another, in particular when these two fuzzy numbers are similar to each other. As a result, defuzzification and ranking of fuzzy numbers are critical problems in fuzzy decision making.

Defuzzication methods have been widely studied for some years and were applied to fuzzy control and fuzzy expert systems $[15,16]$. The major idea behind these methods was to obtain a typical value from a given fuzzy set according to some specified characters, such as central gravity and median. In other words, each defuzzication method provides a correspondence from the set of all fuzzy sets into the set of real numbers [17].

There may be situations where the output of a fuzzy process needs to be a crisp set. Defuzzification is the conversion of a fuzzy quantity to a precise quantity, just as fuzzification is the conversion of a precise quantity to a fuzzy quantity. Among the many methods that have been proposed in recent years, seven are described here for defuzzifying the output of fuzzy functions (membership functions) [18].

In this section we present a new defuzzification method that is used in fitness function of the GA. 
The centroid point of a fuzzy number corresponds to $\bar{x}$ value on the horizontal axis and $\bar{y}$ value on the vertical axis. Suppose $\widetilde{A}_{1}, \widetilde{A}_{2}, \widetilde{A}_{3}, \ldots, \widetilde{A}_{n}$ are triangular fuzzy numbers. First we calculate the gravity center of all numbers (e.g., if $\widetilde{A}_{i}$ is a fuzzy number, the center of its gravity will be $\left.\left(\widetilde{x}_{A_{i}}, \widetilde{y}_{A_{i}}\right)\right)$. We calculate the distance of gravity center of all fuzzy numbers from three points $\left(a_{1}\left(\widetilde{A}_{i}\right), a_{2}\left(\widetilde{A}_{i}\right), a_{3}\left(\widetilde{A}_{i}\right)\right)$ as follows:

$$
\begin{aligned}
& L 1\left(\widetilde{A}_{i}\right)=\sqrt{\left(\tilde{x}_{A_{i}-} a_{1}\left(\widetilde{A}_{i}\right)\right)^{2}+\left(\tilde{y}_{A_{i}}-\mu_{\left(a_{2}\right)}\right)^{2}}, \\
& L 2\left(\widetilde{A}_{i}\right)=\sqrt{\left(\tilde{x}_{A_{i}-} a_{2}\left(\widetilde{A}_{i}\right)\right)^{2}+\left(\tilde{y}_{A_{i}}-\mu_{\left(a_{2}\right)}\right)^{2}}, \\
& L 3\left(\widetilde{A}_{i}\right)=\sqrt{\left(\tilde{x}_{A_{i}-} a_{3}\left(\widetilde{A}_{i}\right)\right)^{2}+\left(\tilde{y}_{A_{i}}-\mu_{\left(a_{2}\right)}\right)^{2}} .
\end{aligned}
$$

Defuzzification value of fuzzy number $\widetilde{A}_{i}$ is calculated via the following equation:

$$
\begin{aligned}
R\left(\widetilde{A_{i}}\right)= & w_{a_{1}} \times L 1\left(\widetilde{A}_{i}\right) \times a_{1}\left(\widetilde{A}_{i}\right)+w_{a_{2}} \times L 2\left(\widetilde{A}_{i}\right) \\
& \times a_{2}\left(\widetilde{A}_{i}\right)+w_{a_{3}} \times L 3\left(\widetilde{A}_{i}\right) \times a_{3}\left(\widetilde{A}_{i}\right) \\
& \times\left(w_{a_{1}} \times L 1\left(\widetilde{A}_{i}\right)+w_{a_{2}} \times L 2\left(\widetilde{A}_{i}\right)\right. \\
& \left.+w_{a_{3}} \times L 3\left(\widetilde{A}_{i}\right)\right)^{-1},
\end{aligned}
$$

where $w_{a_{1}}, w_{a_{2}}$, and $w_{a_{3}}$ are the planner-specified weights. They indicate the relative importance of $a_{1}, a_{2}$, and $a_{3}$ for manager in defuzzification procedure. The values of weight coefficients $w_{a_{1}}, w_{a_{2}}$, and $w_{a_{3}}$ are subjectively selected in the range of $[0,1]$, and they should satisfy the equation $w_{a_{1}}+w_{a_{2}}+w_{a_{3}}=1.0$.

If $\widetilde{A}_{1}, \widetilde{A}_{2}$ are two fuzzy numbers then the ranking will be performed as follows:

$$
\begin{aligned}
& \text { (1) if } R\left(\widetilde{A_{1}}\right)>R\left(\widetilde{A_{2}}\right) \text { then } \widetilde{A}_{1}>\widetilde{A}_{2} \text {; } \\
& \text { (2) if } R\left(\widetilde{A_{1}}\right)=R\left(\widetilde{A_{2}}\right) \text { then } \widetilde{A_{1}} \cong \widetilde{A_{2}} \text {; } \\
& \text { (3) if } R\left(\widetilde{A_{1}}\right)<R\left(\widetilde{A_{2}}\right) \text { then } \widetilde{A_{1}}<\widetilde{A_{2}} \text {. }
\end{aligned}
$$

3.1. Example. Consider the two fuzzy numbers

$$
\begin{aligned}
& \widetilde{A}=(1,2,6), \quad \widetilde{B}=(1,3,6), \\
& \left(w_{A_{1}}=.25, w_{A_{2}}=.5, w_{A_{3}}=.25\right) .
\end{aligned}
$$

By using the proposed method we have

$$
R(\widetilde{A})=3.367, \quad R(\widetilde{B})=3.532, \quad \widetilde{A}(\div) \widetilde{B}=.9534
$$

Hence the ranking order is $\widetilde{B}>\widetilde{A}$.

\section{Problem Formulation}

Notations used for the problem formulation are as follows. $i$ : The number of phases in intersection.

$L_{i}$ : The Lower bound of the green time in phase $i$.

$U_{i}$ : The upper bound of the green time in phase $i$.

TS: The studied period of time.

$\mathrm{TR}_{i}$ : The red time duration in phase $i$.

$\mathrm{TG}_{i}$ : The green time duration in phase $i$.

$\mathrm{CT}_{i}$ : The cycle time duration in phase $i$.

$\mathrm{NCT}_{i}:$ Number of cycles in the TS.

$\widetilde{\mathrm{QR}}_{(i, j)}$ : The fuzzy queue length when red time duration in phase $i$ and $j$ th cycle time ends.

$\widetilde{\mathrm{QG}}_{(i, j)}$ : The fuzzy queue length when green time duration in phase $i$ and $j$ th cycle time ends.

$\widetilde{\mathrm{TIR}}_{i}$ : The fuzzy entrance rate of cars during red time in phase $i$.

$\widetilde{\mathrm{TIG}}_{i}$ : The fuzzy entrance rate of cars during green time in phase $i$.

TOG $\mathrm{G}_{i}$ : The fuzzy exit rate during green time in phase i.

$\mathrm{NTIR}_{i}$ : The fuzzy entrance cars during red time in phase $i$.

$\mathrm{NTIG}_{i}$ : The fuzzy entrance cars during green time in phase $i$.

$\mathrm{NTOG}_{i}$ : The fuzzy exit cars during green time in phase $i$.

$\widetilde{\mathrm{TIP}}_{i}$ : The fuzzy entrance rate of pedestrians during red time in phase $i$.

$\mathrm{NTIP}_{i}:$ The fuzzy entrance pedestrians during red time in phase $i$.

The problem is formulated as the following model:

$\operatorname{Min} Q 1=\sum_{i=1}^{n}\left(\mathrm{NTIR}_{i}+\left(\mathrm{NCT}_{i}-1\right)\right.$

$$
\left.\times\left(\mathrm{NTIR}_{i}+\mathrm{NTIG}_{i}-\mathrm{NTOG}_{i}\right)\right)
$$

$$
\operatorname{Min} Q 2=\sum_{i=1}^{n}\left(\widetilde{\mathrm{TIP}}_{i} \times \mathrm{TR}_{i}\right)
$$


subject to

$$
\begin{gathered}
L_{i} \leq \mathrm{TG}_{i} \leq U_{i}, \\
\mathrm{TR}_{i}=\left(\sum_{i=1}^{n} \mathrm{TG}_{i}\right)-\mathrm{TG}_{i} \quad \forall i, i=1,2, \ldots, n, \\
\mathrm{NTIR}_{i}=\widetilde{\mathrm{TIR}}_{i} \times \mathrm{TR}_{i} \quad \forall i, i=1,2, \ldots, n, \\
\mathrm{NTIG}_{i}=\widetilde{\mathrm{TIG}_{i}} \times \mathrm{TG}_{i} \quad \forall i, i=1,2, \ldots, n, \\
\mathrm{NTOG}_{i}=\widetilde{\mathrm{TOG}_{i}} \times \mathrm{TG}_{i} \quad \forall i, i=1,2, \ldots, n, \\
\mathrm{CT}_{i}=\mathrm{TR}_{i}+\mathrm{TG}_{i} \quad \forall i, i=1,2, \ldots, n, \\
\mathrm{NCT}_{i}=\frac{\mathrm{TS}_{\mathrm{CT}}}{\mathrm{CT}_{i}} \quad \forall i, i=1,2, \ldots, n, \\
\widetilde{\mathrm{QR}}_{(i, j)}=\widetilde{\mathrm{QG}}_{(i, j-1)}+\mathrm{NTIR}_{i} \quad \forall i, i=1,2, \ldots, n .
\end{gathered}
$$

Equation (8) minimizes the total length of traffic queue in all phases of the intersection, and (9) minimizes the total length of pedestrian queue in all phases of the intersection. Equation (10) restricts green time per phase to take a value within a lower bound $L_{i}$ and an upper bound $U_{i}$. Equation (11) represents the red time duration in phase $i$, and (12) represents the fuzzy entrance rate of cars during red time duration in phase $i$.

Equation (13) represents the fuzzy rate of entering cars during green time in Phase $i$. Equation (14) represents the fuzzy rate of leaving cars during green time in phase $i$. Equation (15) shows that the cycle time duration per phase is equal to the sum of green and red time. Equation (16) represents the number of cycle times per phase. The fuzzy queue length when green time duration in phase $i$ and $j$ th cycle times end will be calculated through (16). If the last cycle time duration in the studied period of time (TS) is equal to $m$, the objective function in (8) minimizes the $\widetilde{\mathrm{QR}}_{(i, m)}$.

4.1. Genetic Algorithms for Problem Solving. In this paper, the genetic algorithm is used to optimize the cycle time of the traffic light systems.

4.1.1. Fitness Function. We have fuzzy multiobjective function. In this case, to determine the fitness function $\left(F_{(s)}\right)$ and the probability of selection $\left(P_{(s)}\right)$ for each parent chromosome " $S$ ", initially, we should defuzzify the fuzzy values obtained by (8) and (9) by using the new defuzzification Method described in Section 3. Then, using the following equations, we can determine the $\left(F_{(s)}\right)$ and $\left(P_{(s)}\right)$ :

$$
\begin{gathered}
F_{(s)}=w_{v} \times \frac{Q 1_{s}-Q 1_{\text {min }}}{Q 1_{\text {max }}-Q 1_{\text {min }}}+w_{p} \times \frac{Q 2_{s}-Q 2_{\text {min }}}{Q 2_{\text {max }}-Q 2 C_{\text {min }}}, \\
P_{(s)}=\frac{\left(\sum_{s=1}^{N} F_{(s)}\right) / F_{(s)}}{\sum_{s=1}^{N}\left(\left(\sum_{s=1}^{N} F_{(s)}\right) / F_{(s)}\right)},
\end{gathered}
$$

where $w_{v}$ and $w_{p}$ are the planner-specified weights. They indicate the relative importance of vehicles queue cost and

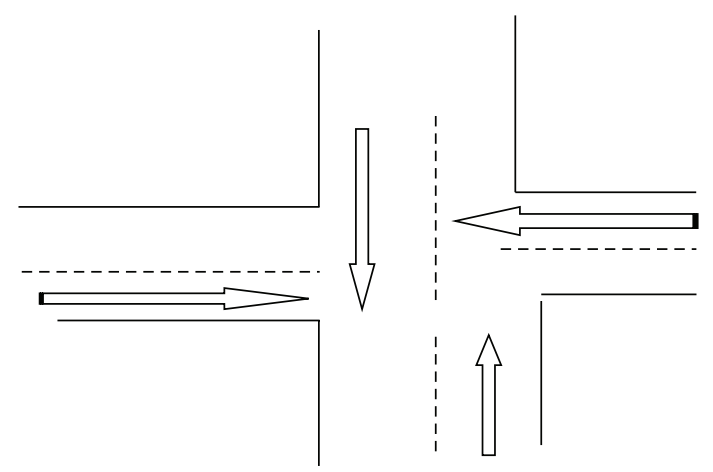

FIGURE 5: An intersection in a traffic network with four phases.

pedestrians' queue cost, respectively. The values of weight coefficients $w_{v}$ and $w_{p}$ are subjectively selected in the range $[0,1]$ by project managers, and they should satisfy the equation $w_{v}+w_{p}=1.0[19]$.

$Q 1_{\max }, Q 1_{\min }, Q 2_{\max }$, and $Q 2_{\min }$ are the maximal and minimal values of the length of traffic and pedestrian queues in the current population. As chromosomes with lower $F_{(s)}$ are more desirable, $P_{(s)}$ should be so defined that the probability of selecting chromosome " $S$ " with lower $F_{(s)}$ is more than other chromosomes. So, equation (19) is introduced for $P_{(s)}$.

4.1.2. Genetic Operators. In this study to produce offsprings from parents for entering the next generation, we used onepoint crossover and one-point mutation. For instance, in an intersection with two phases, two random chromosomes with feasible genes can be as follows:

$$
\begin{aligned}
& \text { Parent } 1=[\mathbf{0}, \mathbf{1}, \mathbf{1}, \mathbf{1}, \mathbf{0}, \mathbf{0}, \mathbf{1}, \mathbf{0}, \mathbf{0}, 1,1,0,0,0] . \\
& \text { Parent } 2=[\mathbf{1}, \mathbf{1}, \mathbf{0}, \mathbf{0}, \mathbf{1}, \mathbf{1}, \mathbf{1}, \mathbf{1}, \mathbf{0}, 0,1,1,0,1] .
\end{aligned}
$$

The offsprings produced from these parents are as follows: ONE-cut-point crossover with random points $\left(e_{1}=9\right)$

$$
\begin{aligned}
& \text { Offspring } 1=[\mathbf{0}, \mathbf{1}, \mathbf{1}, \mathbf{1}, \mathbf{0}, \mathbf{0}, \mathbf{1}, \mathbf{0}, \mathbf{0}, 0,1,1,0,1] . \\
& \text { Offspring } 2=[\mathbf{1}, \mathbf{1}, \mathbf{0}, \mathbf{0}, \mathbf{1}, \mathbf{1}, \mathbf{1}, \mathbf{1}, \mathbf{0}, 1,1,0,0,0] .
\end{aligned}
$$

One-point mutation with random points $\left(e_{1}=6\right)$

$$
\begin{aligned}
& \text { Parent }=[0,1,1,1,0,0,1,0,0,1,1,0,0,0] . \\
& \text { Offspring }=[0,1,1,1,0,1,1,0,0,1,1,0,0,0] .
\end{aligned}
$$

It should be mentioned that the mutation rate $\left(\mathrm{Fm}_{(g)}\right)$ ( $g$ is number of generations) decreases with increasing repetition, (20); so, in the final generation, the mutation rate will be zero:

$$
\operatorname{Fm}_{(g)}=\frac{\operatorname{Fm}_{(g-1)}}{g} .
$$

\section{Illustrative Example}

We considered an intersection, formed by four phases, in a network (see Figure 5).

As an example, an intersection consisting of four phases is presented in this section as depicted in Figure 5. The model 
TABLE 1: The fuzzy entry and exit rates per phase of intersection.

\begin{tabular}{|c|c|c|c|c|}
\hline & $\mathrm{TITR}_{i}$ (vehicle/second) & $\mathrm{TITG}_{i}$ (vehicle/second) & TÖG $_{i}$ (vehicle/second) & $\mathrm{TT}_{i}(\mathrm{man} /$ second $)$ \\
\hline Phase 1 & $(5,7,8)$ & $(5,7,8)$ & $(1,2,3)$ & $(5,6,7)$ \\
\hline Phase 2 & $(2,3,4)$ & $(2,3,4)$ & $(3,4,5)$ & $(11,12,13)$ \\
\hline Phase 3 & $(6,7,8)$ & $(6,7,8)$ & $(6,7,9)$ & $(13,14,15)$ \\
\hline Phase 4 & $(3,4,5)$ & $(3,4,5)$ & $(4,5,6)$ & $(8,10,12)$ \\
\hline
\end{tabular}

TABLE 2: Final outputs of the GA.

\begin{tabular}{ccccccccccc}
\hline$w_{v}$ & $w_{p}$ & $w_{a_{1}}$ & $w_{a_{2}}$ & $w_{a_{3}}$ & $\mathrm{Q} 1$ & $\mathrm{Q} 2$ & $\mathrm{TG}_{1}$ & $\mathrm{TG}_{2}$ & $\mathrm{TG}_{3}$ & $\mathrm{TG}_{4}$ \\
\hline 0.7 & 0.3 & 0.25 & 0.5 & 0.25 & 24563 & 59 & 10 & 15 & 106 & 36 \\
0.8 & 0.2 & 0.25 & 0.5 & 0.25 & 24838 & 59 & 11 & 12 & 120 & 20 \\
0.6 & 0.4 & 0.3 & 0.3 & 0.4 & 25779 & 94 & 16 & 11 & 132 & 22 \\
0.6 & 0.4 & 0.5 & 0.2 & 0.3 & 24002 & 65 & 16 & 14 & 100 & 16 \\
0.5 & 0.5 & 0.3 & 0.4 & 0.3 & 24578 & 60 & 12 & 12 & 120 & 14 \\
0.3 & 0.7 & 0.1 & 0.8 & 0.1 & 25219 & 59 & 10 & 12 & 103 & 39 \\
0.3 & 0.7 & 0.2 & 0.7 & 0.1 & 23719 & 57 & 12 & 11 & 120 & 16 \\
0.2 & 0.8 & 0.4 & 0.2 & 0.4 & 24329 & 59 & 11 & 11 & 128 & 14 \\
0.2 & 0.8 & 0.25 & 0.5 & 0.25 & 25289 & 59 & 10 & 12 & 130 & 59 \\
\hline
\end{tabular}

is programmed in the Microsoft Excel 2007 software, using the Visual Basic Application (VBA). We assumed that the studied period of time (AT) is equal to 30 minutes. In other words, every 30 minutes road detectors send the traffic data to optimization process. We also assumed that the lower bound of the green time duration per phase, $l_{i}$, equals 10 Seconds, and the upper bound of the green time duration per phase, $u_{i}$, equals 138 Seconds. The collected data from the road detectors is shown in Table 1.

In this example, there are than 265 million solutions. The presented model is solved in order to obtain the optimal solution. The planner-specified weights are selected by the manager $\left(\left(w_{v}=0.7, w_{p}=0.3\right)\right.$ and $\left(w_{a_{1}}=.1, w_{a_{2}}=.8, w_{a_{3}}=\right.$ .1)). The GA parameters are set as follows:

$G=100, N=70$, one-point crossover rate $=0.85$, mutation rate $=0.2$.

The output of the proposed algorithm are as: $\mathrm{TG}_{2}=15$, $\mathrm{TG}_{3}=110, \mathrm{TG}_{4}=34$ and its length of traffic and pedestrian queue are $(Q 1=24563, Q 2=59)$ are obtained as the output. The traffic manager may then obtain other optimum solutions by changing the values for the planner-specified weights, which are presented in Table 2 .

\section{Experimental Evaluation}

This section evaluates the performance of our proposed new defuzzification method (NDM) and the centroid point defuzzification method (CPDM) with GA. These algorithms are coded and implemented with the same parameters for twenty-five times. We use the weight relative deviation (WRD) as a common performance measure to compare these algorithms, computed by

$$
\mathrm{WRD}=\left(w_{v} \times \frac{\mathrm{Q} 1_{\mathrm{alg}}-\mathrm{Q} 1_{\text {min }}}{\mathrm{Q} 1_{\text {min }}}+w_{p} \times \frac{\mathrm{Q} 2_{\mathrm{alg}}-\mathrm{Q} 2_{\mathrm{min}}}{\mathrm{Q} 2_{\text {min }}}\right) .
$$
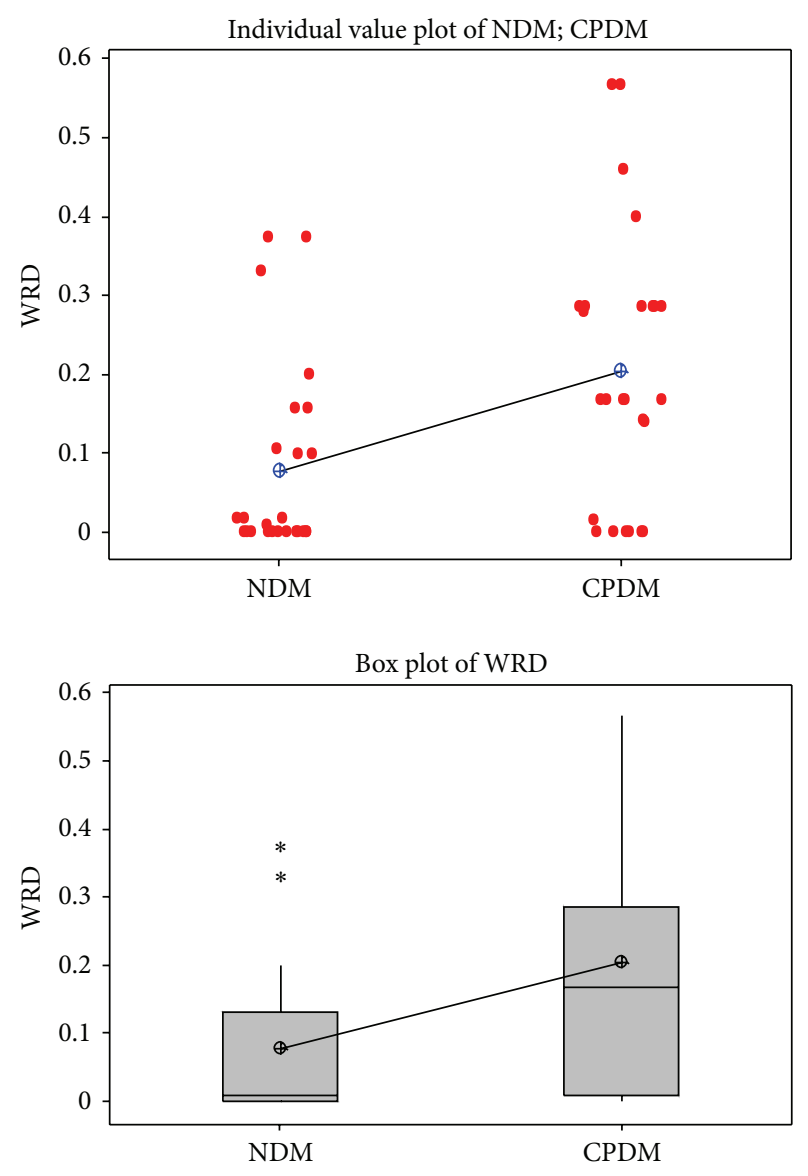

Figure 6: Mean plot and LSD intervals for the NDM and CPDM.

Their results are analysed via the analysis of variance (ANOVA) method. The mean plot and least significant different (LSD) interval for the NDM and CPDM are shown in Figures 6 and 7. It demonstrates that in this problem the NDM gives better outputs than CPDM statistically.

\section{Conclusion}

In this paper, a new multiobjective mathematical model to optimize the timing of traffic light signal of an individual intersection was presented, which minimizes the total traffic queue and pedestrian delays.

Considering the uncertainty in real world, we used fuzzy theory in calculation, and the entrance and exit rates of an intersection were considered as triangular fuzzy numbers. To solve the given problem, we applied genetic algorithm. For more accurate calculation we proposed a new defuzzification 

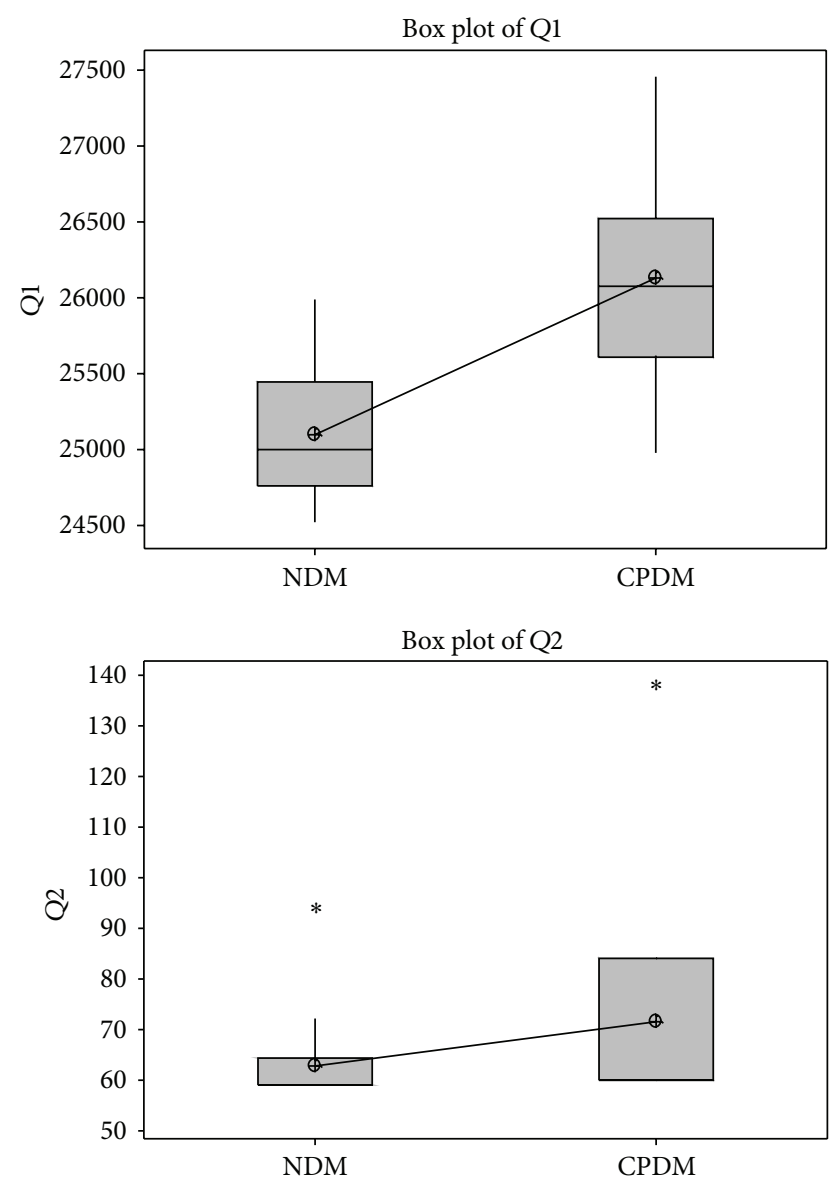

Figure 7: Mean plot of Q1 and Q2 for the NDM and CPDM.

method. The competency of the proposed method was demonstrated, using the weight relative deviation (WRD) measure to compare the performance of the new defuzzification method (NDM) and centroid point defuzzification method (CPDM).

\section{References}

[1] J. M. Bayley, "Pedestrian and traffic control," Traffic Engineering and Control, vol. 8, pp. 311-312, 1966.

[2] R. Noland, "Pedestrian travel times and motor vehicle traffic signals," in Proceedings of the Annual Meeting of the Transportation Research Board, Washington, DC, USA, 1996, paper no. 1553.

[3] R. Noland, "Optimal travel time trade-offs at signalized pedestrian crossings," Unpublished Work, 2003.

[4] E. Brockfeld, R. Barlovic, A. Schadschneider, and M. Schreckenberg, "Optimizing traffic lights in a cellular automaton model for city traffic," Physical Review E, vol. 64, Article ID 056132, 2001.

[5] V. Gradinescu, C. Gorgorin, R. Diaconescu, and V. Cristea, Adaptive traffic light using car-to-car communication [Dissertation], Computer Science Department, University of Bucharest, 2007.

[6] K. T. K. Teo, W. Y. Kow, and Y. K. Chin, Optimization of Traffic Flow within an Urban Traffic Light Intersection with Genetic
Algorithm, School of Engineering and Information Technology, University Malaysia Sabah, Kota Kinabalu, Malaysia, 2010.

[7] F. V. Webster, Traffic Signal Settings, Road Research Technical Paper no. 39, Department of Scientific and Industrial Research, 1958.

[8] Z. Yang, Thesis signal timing optimization based on minimizing vehicle and pedestrian delay by genetic algorithm [Dissertation], Graduate College of the University of Illinois at Urbana-Champaign, 2010.

[9] C. Dong, S. Huang, and X. Liu, "Urban area traffic signal timing optimization based on Sa-PSO," in Proceedings of the International Conference on Artificial Intelligence and Computational Intelligence, vol. 3, pp. 80-84, 2010.

[10] K. K. Tan, M. Khalid, and R. Yusof, "Intelligent traffic lights control by fuzzy logic," Malaysian Jurnal of Computer Science, vol. 3, pp. 45-58, 1996.

[11] J. Favilla, A. Machion, and F. Gomide, "Fuzzy traffic control: adaptive strategies," in Proceedings of the 2nd IEEE International Conference on Fuzzy Systems, pp. 506-511, San Francisco, Calif, USA, March 1993.

[12] M. R. Virkler, Pedestrian Compliance Effects on Signal Delay, Research Record no. 1636, TRB, National Research Council, Washington, DC, USA, 1998.

[13] V. Chilukuri and M. R. Virkler, "Validation of HCM pedestrian delay model for interrupted facilities," Journal of Transportation Engineering, vol. 131, no. 12, pp. 939-945, 2005.

[14] P. Bhattacharya and M. R. Virkler, Optimization for Pedestrian and Vehicular Delay in a Signal Network, Transportation Research Record no. 1939, TRB, National Research Council, Washington, DC, USA, 2005.

[15] D. P. Filev and R. R. Yager, "A generalized defuzzification method via bad distribution," International Journal of Intelligent Systems, vol. 6, pp. 687-697, 1991.

[16] R. R. Yager and D. P. Filev, "SLIDE: a simple adaptive defuzzification method," IEEE Transactions on Fuzzy Systems, vol. 1, no. 1, pp. 69-78, 1993.

[17] L. A. Zadeh, "Fuzzy sets," Information and Control, vol. 8, no. 3, pp. 338-353, 1965.

[18] H. Hellendoorn and C. Thomas, "Defuzzification if fuzzy controllers," Journal of Intelligent Fuzzy Systems, vol. 1, pp. 109-123, 1993.

[19] N. Shahsavari-Pour, M. Modarres, R. Tavakoli-Moghadam, and E. Najafi, "Optimizing a multi-objectives time-cost-quality trade-off problem by a new hybrid genetic algorithm," World Applied Science Journal, vol. 10, no. 3, pp. 355-363, 2010. 


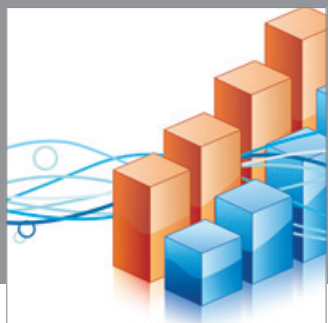

Advances in

Operations Research

mansans

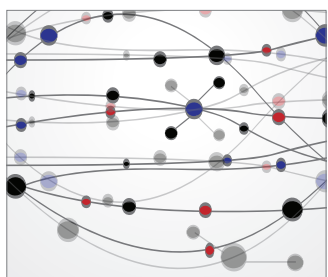

The Scientific World Journal
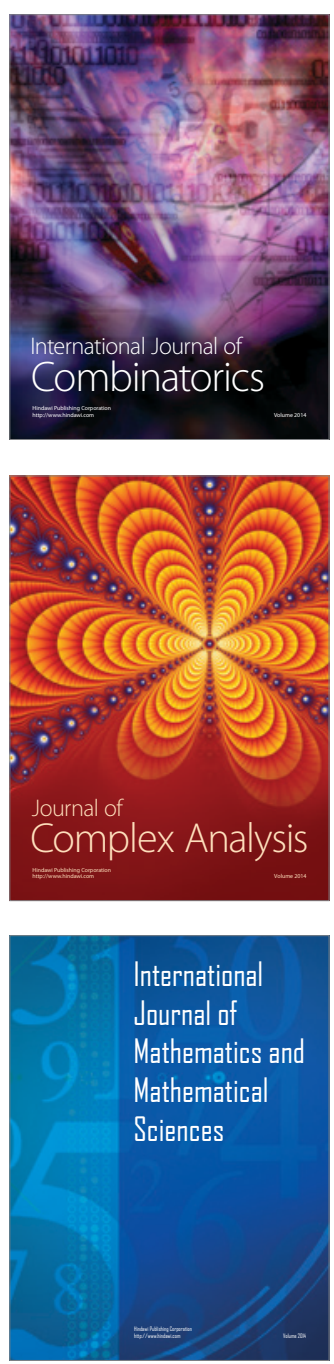
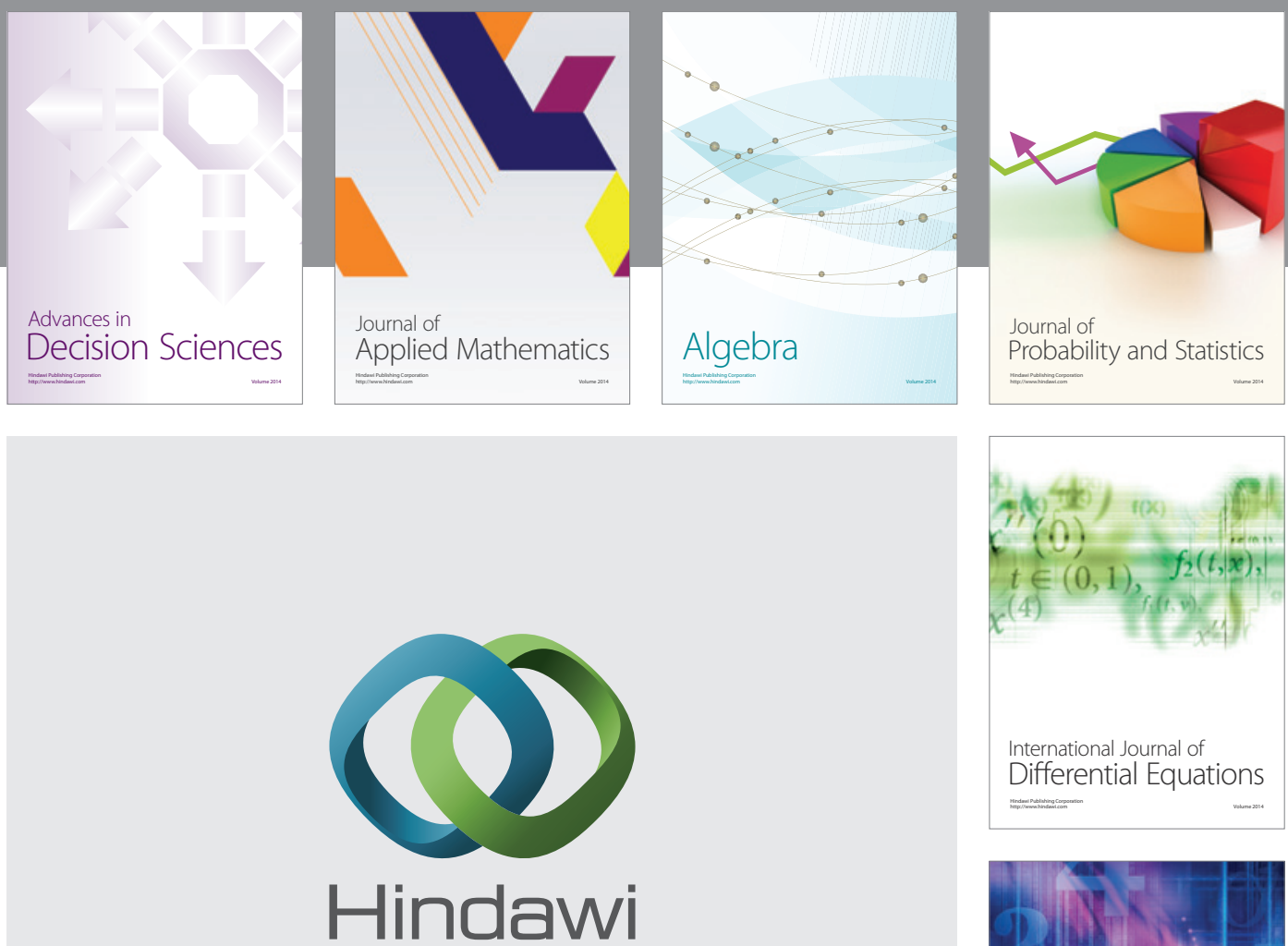

Submit your manuscripts at http://www.hindawi.com
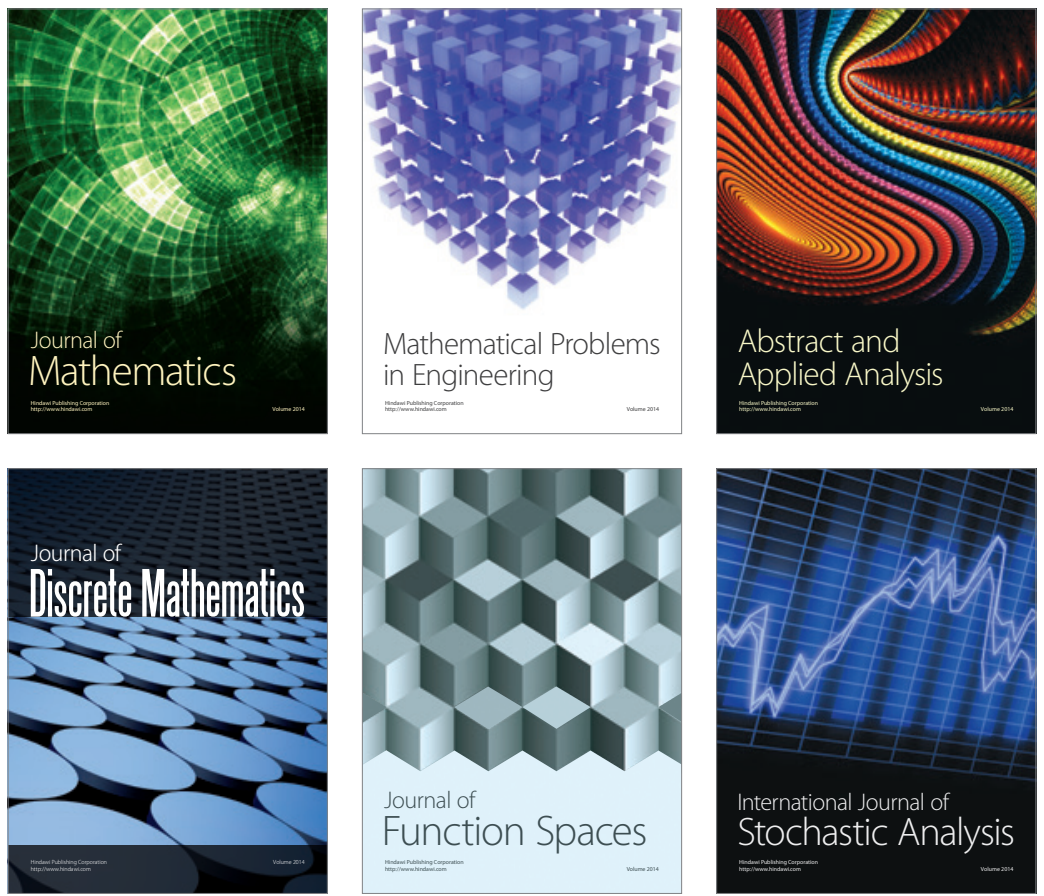

Journal of

Function Spaces

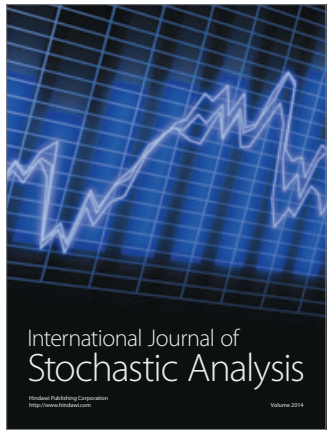

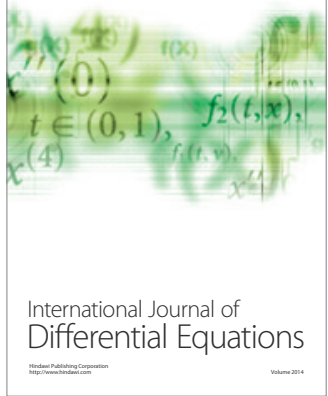
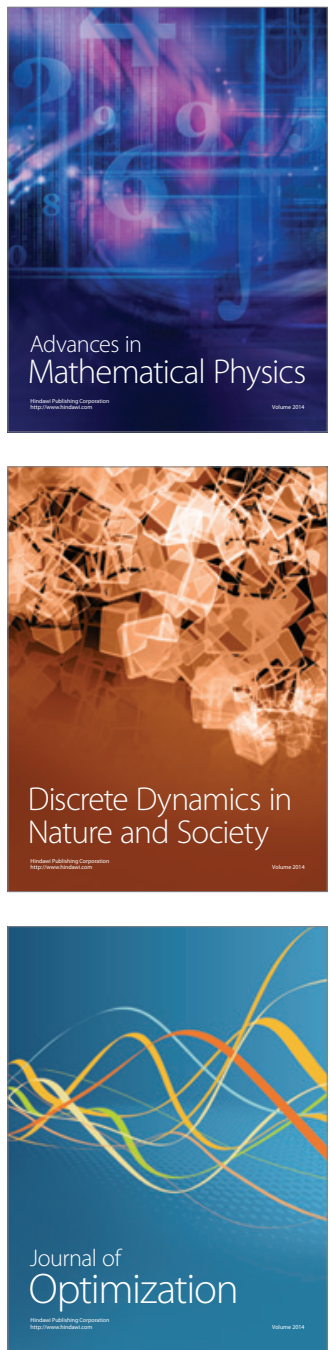